\title{
Gerenciamento de enfermagem em sala de vacina: desafios e potencialidades
}

Nursing management in the vaccine room: challenges and potentialities

Gerencia de enfermería en sala de vacuna: retos y potencialidades

\section{Matheus Adriano Divino Pereira ${ }^{\mathrm{I}}$, Bruna Camargos de Lima ${ }^{\mathrm{II}}$, Deborah Amaral Donnini ${ }^{\mathrm{III}}$, Valéria Conceição de Oliveira ${ }^{\mathrm{IV}}$, Tarcísio Laerte Gontijo ${ }^{\mathrm{v}}$, Heloiza Maria Siqueira Rennovi}

\begin{abstract}
Resumo: Objetivo: analisar o gerenciamento de enfermagem em sala de vacina, com ênfase na supervisão, em um município de médio porte de Minas Gerais, Brasil. Método: estudo descritivo, de abordagem qualitativa, tipo estudo de caso único. Os dados foram organizados e analisados por meio da Análise de Conteúdo modalidade temático-categorial. Resultados: os achados foram organizados em duas categorias analíticas: "Fatores intervenientes no gerenciamento em sala de vacina", abordando desafios para supervisão, necessidade de um instrumento que padronize essa atividade, falta de organização do processo de trabalho e sobrecarga de funções. A segunda categoria: "Potencialidades para o gerenciamento na sala de vacina" aponta como fatores potencializadores a organização do tempo e agenda do enfermeiro e a necessidade de um instrumento que sistematize as atividades de supervisão. Considerações finais: a maioria dos enfermeiros compreendem a importância da supervisão, como ferramenta do gerenciamento, mas não a realizam de forma sistemática.

Descritores: Enfermagem; Gestão em Saúde; Supervisão de enfermagem; Organização e administração; Vacinas
\end{abstract}

Abstract: Objective: to analyze nursing management in the vaccine room, with emphasis on supervision, in a medium-sized city of Minas Gerais, Brazil. Method: a descriptive, qualitative, unique case study. The data were organized and analyzed through theme/category-based content analysis. Results: the findings were organized into two analytical categories: "Intervening factors in the management of the vaccine room", addressing the challenges for supervision, the need for an instrument to standardize this activity, lack of work process organization and

\footnotetext{
${ }^{\text {I }}$ Enfermeiro. Mestrando em Enfermagem pela Universidade Federal de São João del-Rei - UFSJ/Campus Centro Oeste. Divinópolis, MG, Brasil. E-mail: mth.adri@gmail.com. ORCID: https://orcid.org/0000-0002-5127-5659

II Enfermeira. Residente do Programa de Residência em Enfermagem Obstétrica do Hospital Sofia Feldman. Belo Horizonte, MG, Brasil. E-mail: buhcamargos@hotmail.com. ORCID: https://orcid.org/0000-0001-6654-5618

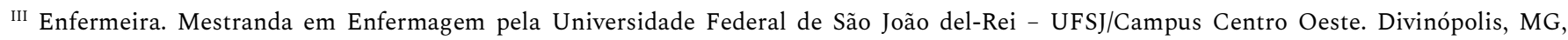
Brasil. E-mail: deborahdonnini@hotmail.com. ORCID: https://orcid.org/0000-0003-1238-7524

IV Enfermeira. Doutora em Enfermagem. Professora na Universidade Federal de São João del-Rei - UFSJ/Campus Centro Oeste. Divinópolis, MG, Brasil. E-mail: valeria.oli.enf@gmail.com. ORCID: http://orcid.org/0000-0003-2606-9754

v Enfermeiro. Doutor em Ciências da Saúde. Professor na Universidade Federal de São João del-Rei - UFSJ/Campus Centro Oeste. Divinópolis, MG, Brasil. E-mail: enftarcisio@ufsj.edu.br. ORCID: https://orcid.org/0000-0001-7845-3825

${ }^{V I}$ Enfermeira. Doutora em Enfermagem. Professora na Universidade Federal de São João del-Rei - UFSJ/Campus Centro Oeste. Divinópolis, MG, Brasil. E-mail: heloizarenno@gmail.com. ORCID: https://orcid.org/0000-0002-8142-9313
} 
overloading of functions. The second category: "Potentialities for the management in the vaccine room" points out as potentiating factors the organization of the nurse's time and agenda and the need for an instrument that systematizes the supervision activities. Final Remarks: Most nurses understand the importance of supervision as a management tool, but do not perform it systematically.

Descriptors: Nursing; Health Management; Nursing, Supervisory; Organization and administration; Vaccines

Resumen: Objetivo: analizar la gerencia de enfermería en sala de vacuna, con énfasis en supervisión, en un municipio de tamaño mediano en Minas Gerais, Brasil. Método: estudio descriptivo, cualitativo, tipo estudio de caso único. Los datos fueron organizados y analizados a través de modalidad temática y categoría de Análisis de Contenido. Resultados: los hallazgos se organizaron en dos categorías analíticas: "Factores que intervienen en la gerencia en sala de vacuna", que abordan desafíos de la supervisión, necesidad de instrumento para estandarizar esta actividad, falta de organización del trabajo y sobrecarga de funciones. La segunda categoría: "Potencialidades para gerencia en sala de vacuna" señala factores potenciadores en organización del tiempo y agenda de la enfermera y la necesidad de un instrumento que sistematice las actividades de supervisión. Consideraciones finales: la mayoría de las enfermeras entienden la importancia de la supervisión como una herramienta de gestión, pero no realizan de manera sistemática.

Descriptores: Enfermería; Gestión en salud; Supervisión de Enfermería; Organización y Administración; Vacunas

\section{Introdução}

A imunização constitui uma das medidas mais eficazes na prevenção de doenças e requer conhecimento adequado que garanta sua qualidade. ${ }^{1}$ A Organização Mundial da Saúde (OMS) destaca que um programa de vacinação eficiente tem benefícios para a saúde e conclui que a imunização tem a capacidade de alcançar amplos ganhos sociais e/ou comunitários, mais facilmente do que qualquer outra intervenção em saúde. ${ }^{2}$

O sucesso da vacinação não deve considerar apenas o cumprimento da cobertura vacinal, e sim das condições ideais de armazenamento, preparo e administração dessas vacinas. ${ }^{1}$ Nesse contexto, o enfermeiro se torna um importante personagem, realizando a gestão de enfermagem na produção do cuidado, direcionando o serviço para o alcance dos resultados esperados e repercutindo benéfica e satisfatoriamente na qualidade da assistência prestada. ${ }^{3}$

No Brasil, o Ministério da Saúde, com o intuito de garantir o manuseio, conservação e administração dos imunobiológicos de forma correta, instituiu o Programa Nacional de Imunização (PNI), recomendando que as atividades em sala de vacina sejam realizadas por uma equipe de enfermagem capacitada, sendo o enfermeiro o responsável pela supervisão. ${ }^{4} \mathrm{~A}$ sala 
precisa ser utilizada pelo profissional como uma ferramenta gerencial do cuidado, no preparo de pessoal e aperfeiçoamento constante para atingir êxito na qualidade da assistência. ${ }^{5}$

O PNI é estratégia prioritária no controle e erradicação de doenças imunopreveníveis na atualidade. Destaca-se que as Unidades Básicas de Saúde (UBS) são estruturas fundamentais para o desenvolvimento desse Programa, realizando a maior parte da vacinação no território nacional e demonstrando, assim, que elas exercem papel importante na prevenção de doenças, sendo porta principal de entrada para o Sistema Único de Saúde (SUS), conforme recomendado pela Política Nacional de Atenção Básica (PNAB). ${ }^{6-7}$

Em um estudo realizado em Minas Gerais, para compreender a percepção do enfermeiro sobre a supervisão das atividades realizadas em sala de vacina, identificaram-se diversas dificuldades para desenvolvê-la. ${ }^{8}$ Por isso, faz-se necessário demonstrar sua importância na sala de vacina, não se limitando apenas à supervisão do trabalho da unidade de saúde, como também a necessidade de atender às diretrizes estabelecidas pelo PNI. ${ }^{6}$

O enfermeiro, como gestor do cuidado, precisa vislumbrar que a supervisão em sala de vacina tem um impacto social relevante, fazendo-se necessária a superação das dificuldades que possam interferir nessa atividade e prejudicar o seu verdadeiro significado para os envolvidos no processo.

Partindo do pressuposto de que o enfermeiro é o responsável pelo gerenciamento do cuidado em sala de vacina e de que a supervisão de enfermagem é uma importante ferramenta para a qualidade da assistência, pergunta-se: como acontece a prática de supervisão em enfermagem no gerenciamento de salas de vacina?

Assim, este trabalho busca analisar o gerenciamento de enfermagem em sala de vacina, com ênfase na supervisão, em um município de médio porte de Minas Gerais, Brasil.

\section{Método}


Gerenciamento de enfermagem em sala de vacina: desafios e potencialidades $\mid 4$

Trata-se de um estudo descritivo, de abordagem qualitativa, tipo estudo de caso único. ${ }^{9} \mathrm{O}$ caso em estudo é a região Sudeste de saúde em um município de médio porte do centro-oeste de Minas Gerais que conta com uma população estimada em 232.945 mil habitantes. ${ }^{10}$ Essa região compõe-se de nove UBS, em que duas são classificadas como Centro de Saúde (CS) e sete como Estratégia Saúde da Família (ESF).

Cada CS possui em sua equipe dois enfermeiros, sendo um deles o Responsável Técnico (RT) pela equipe de enfermagem. Cada ESF possui um enfermeiro que, por conseguinte, é o RT de sua UBS. A Referência Técnica em Imunização encontra-se na Secretaria Municipal de Saúde do município, gerenciando o serviço.

O estudo de caso engloba a abordagem qualitativa, apontando um fenômeno contemporâneo, no seu contexto real. A sua caracterização se dá pela utilização de múltiplos métodos de coleta de dados, com o intuito de conhecer o fenômeno nas suas várias faces. ${ }^{9}$ Nesse sentido, a utilização do estudo de caso único representa uma narrativa simples para descrever e analisar o contexto, tendo em vista sua capacidade de lidar com uma quantidade de evidências documentos, artefatos, entrevistas e observações -, além do que pode estar disponível no estudo histórico convencional. ${ }^{9}$

O caso analisado foi a região Sudeste do município e as unidades de análise foram as nove UBS existentes nessa região. A escolha se deu por indicação da coordenação do serviço de Referência Técnica em Imunização da Secretaria Municipal de Saúde, devido a fragilidades da manutenção da cadeia de frio. Os participantes foram os enfermeiros RT de cada Unidade de Saúde. Os critérios de inclusão foram: ser enfermeiro em atividade na rede básica de saúde do município alocado na UBS e ser responsável pela supervisão da sala de vacina. Foram excluídos os enfermeiros que estavam de férias, licença saúde ou afastados. Atendendo a esses critérios, de 11 enfermeiros, nove foram elegíveis para o estudo. 
5 IPereira MAD, Lima BC, Donnini DA, Oliveira VC, Gontijo TL, Renno HMS

Para atender os pressupostos apresentados, a pesquisa procurou, durante a coleta de dados, evidências convergentes ou divergentes com respeito aos fatos e às conclusões, tendo como fonte de evidências uma entrevista semiestruturada com os enfermeiros das UBS responsáveis pela supervisão nas salas de vacina e uma observação direta não participante nas salas de vacina com registro em diário de campo.

Foi realizado contato telefônico com os enfermeiros das Unidades de Saúde para o agendamento prévio das entrevistas e observações. Todos os nove enfermeiros aceitaram participar do estudo, em acordo com Termo de Consentimento Livre e Esclarecido (TCLE).

A coleta de dados ocorreu no período de agosto a novembro de 2017, por três pesquisadores previamente treinados. As entrevistas e as observações foram realizadas na própria UBS onde o enfermeiro estava inserido, guiadas por um roteiro norteador. As entrevistas duraram em média 30 minutos e foram gravadas em arquivo digital. Posteriormente, foram transcritas literalmente, preservando-se a fidedignidade das informações.

O roteiro semiestruturado abordou aspectos da atividade de gerenciamento em sala de vacina pelo enfermeiro, com enfoque na supervisão, ou seja: percepção do enfermeiro sobre a supervisão em sala de vacina; fatores que interferem na supervisão; instrumentos utilizados para a supervisão; percepção sobre a supervisão da sala de vacina na qualidade da assistência prestada; possíveis estratégias para a melhoria da supervisão em sala de vacina. Os discursos referentes às entrevistas estão identificados com a letra E, correspondente ao enfermeiro, seguida dos números de um a nove, sequencialmente.

A observação direta foi realizada durante cinco dias na sala de vacina de cada unidade, por apenas uma pessoa. O roteiro para a realização da observação direta abordou: a presença do enfermeiro na sala de vacina; identificação das atividades de supervisão; identificação dos instrumentos de supervisão utilizados pelo enfermeiro e identificação dos fatores que facilitam 
ou dificultam a supervisão do enfermeiro em sala de vacina. O registro dessas informações foi feito em um diário de campo, elaborado a cada período, com duração média de quatro horas, identificado como "Notas de Observação" (NO).

Para avaliação crítica dos dados utilizou-se a análise de conteúdo modalidade temáticocategorial, ${ }^{11}$ com o auxílio dos recursos do software Atlas.ti 8.0, ${ }^{12}$ considerando o objetivo do estudo; por meio da exploração do material empírico, com leitura exaustiva dos registros, separação dos recortes de acordo com as convergências, divergências, classificação e agregação dos dados em temas.

A pesquisa atendeu todos os preceitos éticos descritos na resolução no 466/2012 do Conselho Nacional de Saúde, a qual compreende aspectos que devem ser atendidos diante de pesquisas realizadas com seres humanos, ${ }^{13}$ e foi aprovada pelo Comitê de Ética em Pesquisa da Universidade Federal de São João del-Rei - Campus Centro-Oeste, através do parecer de $\mathrm{n}^{\circ}$ 2.168.374, datado de 11 de julho de 2017.

\section{Resultados e discussão}

Todos os participantes do estudo eram do sexo feminino e com idade média de 37 anos, variando entre 29 e 45 anos. Observou-se que todas as enfermeiras exerciam as atividades de RT na UBS há mais de um ano. A partir da análise dos dados obtidos, emergiram duas categorias analíticas: a) Fatores intervenientes no gerenciamento em sala de vacina; e b) Potencialidades para o gerenciamento na sala de vacina.

\section{Fatores intervenientes no gerenciamento em sala de vacina}


7 IPereira MAD, Lima BC, Donnini DA, Oliveira VC, Gontijo TL, Renno HMS

A formação do enfermeiro voltada para a supervisão em sala de vacina apareceu nessa categoria como um importante discurso dos profissionais, que relataram a existência e déficit nesse aspecto, influenciando, de forma negativa, a supervisão.

Eu não tinha ouvido falar que o enfermeiro tinha que fazer supervisão em sala de vacina na época que eu formei. (E1)

Muitas vezes a formação do enfermeiro voltada para a sala de vacina é muito defasada, o que dificulta muito quando começamos a trabalhar. (E2)

A supervisão em sala de vacina não é uma atividade frequente nos serviços públicos de saúde no Brasil, demonstrando por vezes a existência de pouco conhecimento sobre a temática pelos profissionais. Por conseguinte, a deficiência da supervisão de enfermagem pode comprometer a qualidade dos imunobiológicos ofertados à população e, consequentemente, comprometer a qualidade da assistência. ${ }^{14}$

Entre os entrevistados, dois citaram a falta de conhecimento do enfermeiro sobre a supervisão em sala de vacina, relatando um conceito de supervisão reducionista. Em geral, no cotidiano das Unidades de Saúde, foi possível observar que o enfermeiro comparecia na sala de vacina esporadicamente, apenas quando a demanda de suas atividades no serviço era pequena, para recolher os mapas de administração de imunobiológicos e utilizar o computador para outras funções. (NO)

Provavelmente isso aconteça devido às lacunas de formação do enfermeiro, tais como comunicação em uma perspectiva participativa, dialógica, relacionamento interpessoal, gerenciamento de conflitos e do próprio exercício da supervisão ${ }^{3}$ ou até mesmo a desvalorização dessa atividade, o que descaracteriza o seu verdadeiro papel de supervisor em sala de vacina.

A formação, portanto, ainda não se encontra em sintonia com as diversas demandas do SUS, incluindo a supervisão. ${ }^{15}$ Nesse sentido, estudo aponta que mediante a qualificação dos 
Gerenciamento de enfermagem em sala de vacina: desafios e potencialidades I 8

profissionais o setor da saúde irá se tornar um lugar de atuação crítica, reflexiva e tecnicamente competente. ${ }^{16}$

A falta de sistematização do trabalho do enfermeiro aparece em geral, influenciando negativamente o processo de supervisão. Isso pode ser observado nos depoimentos a seguir.

Ah, então até quando não tem vacina para fazer, eu fico lá observando, fico perguntando, fico como quem não quer nada. (E2)

Às vezes, eu vou lá para dar uma olhada, faço alguma vacina e olho os mapas de temperatura. (E8)

A falta de planejamento e a dificuldade por parte do enfermeiro em explicitar como acontece o processo de supervisão em sala de vacina fazem com que o profissional não priorize essa atividade em seu cotidiano de trabalho. Isso corrobora para que a supervisão seja assistemática e pouco planejada, sinalizando ausência do cumprimento dos objetivos propostos pela supervisão, que incluem planejamento, organização e avaliação do serviço e a educação permanente dos trabalhadores. ${ }^{17}$

Distante das atividades da sala de vacina, o enfermeiro não consegue identificar as demandas e necessidades de sua equipe, o que acaba comprometendo o planejamento do cuidado, da supervisão e da orientação na perspectiva do acompanhamento do trabalho da equipe. ${ }^{6}$ Nesse sentido, o enfermeiro necessita apropriar-se de conhecimentos científicos, teóricos e práticos em supervisão de enfermagem, para que o desempenho dessa atribuição seja alcançado com excelência. ${ }^{3}$

Em consonância com esse achado, os relatos estão relacionados às múltiplas atividades e funções que o enfermeiro desempenha dentro da UBS. O excesso de atividades relatado referese àquelas específicas do enfermeiro e às ações de responsabilidade de outros profissionais.

Mas é questão da demanda mesmo da população, muito serviço burocrático, muita papelada, muita coisa que a gente tem que fazer, tem hora que a gente vai deixando 
algumas coisas à parte. Então acho que seria mais isso, pela sobrecarga mesmo que o enfermeiro tem. (E1)

Recursos Humanos insuficiente, em que a gente tem que realizar funções que nem cabe à gente, do enfermeiro. (E7)

Tais relatos convergem com os achados de estudo realizado no Brasil com 11 enfermeiros de UBS, que visou entender como ocorre a prática gerencial do enfermeiro nessas unidades. ${ }^{18}$ Nessa pesquisa, identificou-se que a sobrecarga de trabalho está relacionada ao acúmulo de funções administrativas e assistenciais, além da realização de funções não específicas.

A supervisão necessita de tempo, planejamento e priorização para ser posta em prática no processo de trabalho do enfermeiro. Porém, é preciso considerar que ainda existe a prevalência do modelo em que as atividades, chamadas de ações curativas, são priorizadas a despeito das atividades ligadas às ações preventivas, corroborando com os achados deste estudo. ${ }^{8}$

O trabalho do enfermeiro necessita ser sistematizado em todas as suas fases, ou seja, desde a escolha das intervenções até a avaliação dos resultados, incluindo o planejamento, a organização e a realização cotidiana do processo de trabalho. ${ }^{14}$

A partir das observações, ficou evidente que a maioria dos enfermeiros, de fato, passam a maior parte do tempo realizando serviços burocráticos, como o lançamento de produção, encaminhamentos, dentre outros, o que dificulta a supervisão em sala de vacina. Além dos recursos humanos, outros fatores já apontados são a quantidade insuficiente dos insumos. (NO)

Também dificulta bastante a supervisão em sala de vacina a falta de insumos, todos os insumos, não só vacina. Então, a falta do técnico, computador, vacina, impresso também dificulta, e por aí vai. (E2)

A falta desses insumos também foi observada, em algumas unidades, em que coexistiam a falta de vacinas (rotavírus), a falta de computador exclusivo para a sala de vacina e a falta de agulhas específicas, por exemplo. (NO)

As UBS necessitam dispor de instalações físicas adequadas para as atividades de vacinação de acordo com a Resolução da Diretoria Colegiada - RDC n ${ }^{\circ} 50$, de 21 de fevereiro de 
2002, ou regulamentação que venha a substituí-la. ${ }^{19}$ Com isso, oferece um ambiente físico mais adequado para o desempenho das tarefas laborais, diminuindo a exposição dos profissionais a potenciais fontes de riscos ocupacionais e melhorando a qualidade do atendimento prestado. ${ }^{18,20}$

Quando existe a divergência entre os recursos materiais, físicos e/ou humanos, é passível de se gerar uma situação complicadora para os trabalhadores, capaz de aumentar a tensão no ambiente de trabalho - o que desmotiva e interfere na qualidade da assistência. É preciso que a estrutura oferecida pela UBS possa conformar, no espaço, todas as suas necessidades organizativas, assistenciais e da comunidade. ${ }^{18,21}$

Para além do quantitativo de recursos e insumos, surge a resistência dos técnicos de enfermagem em se adequar a supervisão do enfermeiro na sala de vacina, como foi relatado nos depoimentos.

Outro fator que dificulta a supervisão do enfermeiro em sala de vacina são os profissionais que atuam na sala; muitas vezes eles colocam empecilhos, são resistentes. (E2)

Quando o técnico de enfermagem que está responsável pelas vacinas não acata as condutas, os protocolos que têm que ser seguidos da sala são muito complicados. (E9)

Eles querem fazer da forma que eles acham que é o certo, ou que eles aprenderam daquela forma e não aceitam mudanças. (E3)

Percebe-se, diante das entrevistas, que o profissional atuante na sala de vacina muitas vezes não se adapta às cobranças de suas atribuições. O supervisor, por outro lado, reconhece no comportamento dos profissionais essa resistência e julga-a como algo prejudicial à relação interna da equipe, o que traz consequências ao relacionamento interpessoal dos trabalhadores. ${ }^{22}$

Essa instabilidade pode tornar o ambiente de trabalho negativo, interferindo no cuidado destinado aos pacientes, ficando sem interação, formal e rotineiro, mecanizando a assistência. Logo, o cuidado destinado ao paciente passa a ser prejudicado, pois a assistência acaba sendo permeada por fatores negativos, como a insatisfação e desgaste emocional dos profissionais. ${ }^{22}$ 
11 IPereira MAD, Lima BC, Donnini DA, Oliveira VC, Gontijo TL, Renno HMS

Nesse contexto, o fortalecimento das relações interpessoais na equipe de enfermagem precisa alcançar uma consolidação democrática da profissão. É necessária uma atenção sobre a ação comunicativa durante o processo de trabalho da enfermagem, permitindo qualificar o relacionamento interpessoal, com autonomia de seus agentes e construção mútua de objetivos comuns na equipe. Por fim, os componentes desse processo interativo precisam se reconhecer como atores de um mundo social repleto de divergentes culturais, saberes e subjetividades, para possibilitar o relacionamento eficaz ${ }^{22}$ e considerar o aspecto de reciprocidade presente na supervisão., 23

\section{Potencialidades para o gerenciamento na sala de vacina}

Em relação aos fatores que potencializam o gerenciamento, com ênfase na supervisão em sala de vacina, os enfermeiros entrevistados destacaram a organização do processo de trabalho como fundamental para melhorar a qualidade do serviço.

Então eu acho que é questão mesmo de organização. $O$ enfermeiro tem que se empoderar e ver isso como função dele de organizar, não é só deixar e ir fazendo vacina, ele tem que fazer, sim, a função de supervisão. (E6)

A maioria dos entrevistados também relatou a própria atribuição de realizar a supervisão como forma de melhorar o cuidado prestado.

Acho que se tem supervisão boa em sala de vacina, significa que tudo vai funcionar bem lá dentro. Isso vai influenciar na qualidade da assistência e na confiança da população com a gente também. Menos problemas e mais confiança, mais benefícios, sem dúvidas disso. (E3)

Isso ressalta a importância da supervisão de enfermagem como instrumento gerencial, que pode facilitar o planejamento, a implementação, avaliação do cuidado e a orientação do trabalho em equipe ${ }^{3}$, bem como, consequentemente, favorecer a qualidade do serviço prestado, busca de estratégias em soluções de problemas e, inclusive, promover propostas educativas e relacionais com os demais membros da equipe. ${ }^{24}$ 
Em estudo ${ }^{25}$ foi observado que, além da organização do processo de trabalho, como fator potencializador de ambiente organizado e adequado, ofertando bom dimensionamento de recursos humanos e satisfação profissional, existe o sentimento de alta exigência para alcançar as metas institucionais. Isso mostra cada vez mais autonomia sobre o instituído, reconhecendo fatores positivos da organização do serviço, sem deixar de apontar as questões que geram desgaste e desconforto. ${ }^{25}$

Nesse sentido, a supervisão considerada como ferramenta pode nortear o serviço do enfermeiro no alcance de resultados desejáveis, beneficiando satisfatoriamente a organização do processo de trabalho. A adoção do Processo de Enfermagem representa uma forma de se concretizar essa lógica, possibilitando a organização e condução do trabalho, a partir de um instrumento próprio da profissão. ${ }^{25}$

A necessidade de um instrumento de gestão pode ser considerada outro fator importante para nortear o planejamento da atividade do enfermeiro e melhorar a qualidade no cuidado, com o intuito de sistematizar e coordenar a supervisão em sala de vacina, como evidenciado a seguir.

Eu acho que seria interessante existir um instrumento de supervisão. Eu acho que, que é uma supervisão meio empírica, não tem, não é sistemática. (E8)

Seria importante um instrumento específico. (E7)

Foi possível observar que, na maioria das vezes, os enfermeiros, ao entrarem na sala de vacina, não seguiam um padrão para realizar a supervisão. Eles apenas conferiam alguns materiais e temperatura da câmara fria. (NO)

Pode-se destacar que as técnicas e instrumentos de supervisão que são utilizados pelos enfermeiros no desenvolvimento do seu trabalho compõem-se de pouca ou nenhuma sistematização, mesmo reconhecendo sua relevância no processo de trabalho, de forma específica, na supervisão. ${ }^{26}$ 
O PNI disponibiliza, desde 2004, o Programa de Avaliação do Instrumento de Supervisão em Sala de Vacinação (PAISSV), utilizado por coordenadores estaduais de imunizações para padronizar o perfil de avaliação e a agilidade na tabulação dos resultados encontrados. ${ }^{27}$ Porém, ressalta-se que, no limite de conhecimento desses autores, não há na literatura instrumentos sistematizados e validados para a prática de supervisão em enfermagem em salas de vacina, específicos para uso pelo enfermeiro. Ou seja, as concepções sobre supervisão ficam marcadas por contradições, sendo traduzidas, na prática concreta, por uma supervisão desarticulada e fragmentada. ${ }^{28}$

Infere-se que esta pesquisa apresenta limitações quanto ao seu desenvolvimento, abarcando somente as UBS da região Sudeste do município, além de apresentar um número reduzido de Unidades, permitindo considerar os resultados encontrados apenas para a região estudada. No entanto, as reflexões e as observações que emanaram aprofundam a compreensão de como vem ocorrendo a supervisão da sala de vacina e abre a perspectiva para futuros trabalhos de temáticas como a criação de um instrumento de supervisão, a análise do processo ensino-aprendizagem do enfermeiro, dentre outros.

\section{Considerações finais}

A construção do conhecimento acerca da prática gerencial do enfermeiro no âmbito das UBS é imprescindível no sentido de tomar decisões e inovar nas atividades desenvolvidas com a equipe e a comunidade. Destaca-se, ainda, a supervisão como forma de auxílio nessa prática, a fim de prestar um atendimento qualificado e resolutivo à população.

O estudo apontou que a maioria dos enfermeiros entende a importância da supervisão em sala de vacina como uma ferramenta gerencial, mas não a realiza de forma efetiva. Tal fator é influenciado pela falta de organização do processo de trabalho, sobrecarga de funções e inexistência de um instrumento que padronize essa atividade. 
Além disso, a ausência de orientações sobre como realizar a supervisão também dificulta a atuação do enfermeiro em sala de vacina. Isso evidencia a relevância de investimentos na formação acadêmica dos profissionais e aperfeiçoamento dos enfermeiros, por meio de medidas como a educação permanente sobre a supervisão em sala de vacina, incluída como ferramenta no seu processo de trabalho.

A organização do tempo e agenda do enfermeiro como fator facilitador do processo de supervisão foi destacado pelos participantes. A partir dos registros em diário de campo, ficou evidente que o enfermeiro que organiza e planeja suas atividades, consegue realizar a supervisão em sala de vacina de forma efetiva. Por conseguinte, a criação de um instrumento que sistematize esse processo, padronizando o direcionamento da supervisão pelo enfermeiro, possibilita respaldo profissional por meio dos registros e a continuidade/complementaridade do cuidado prestado com qualidade.

Portanto, o desafio para o enfermeiro é repensar o seu processo de trabalho e desenvolver estratégias para realizar a supervisão em sala de vacina de maneira eficaz, a fim de garantir a qualidade do cuidado prestado.

\section{Referências}

1. Marinelli NP, Carvalho KM, Araújo TME. Conhecimento dos profissionais de enfermagem em sala de vacina: análise da produção científica. RevUnivap.[Internet]2015[acesso em 2017 ago 21]; 21(38):26-35. Disponível em: https://revista.univap.br/index.php/revistaunivap/article/view/324/293 doi: 10.18066/revistaunivap.v21i38.324

2. Doherty M, Buchy P, Standaert B, Giaquinto C, Prado-Cohrs D. Vaccine impact: benefits for human health. Vaccine.[Internet] 2016[acesso em 2017 set 03];34(52): 6707-14. Disponível em: https://ac.elscdn.com/S0264410X16309434/1-s2.0-S0264410X16309434-main.pdf?_tid=af34516d-3edd-462b-b8ef3211e77c4939\&acdnat=1541639016_44c69540bb43e2327877e004f8440f64 doi: 10.1016/j.vaccine.2016.10.025.

3. ChavesLDP, MininelVA, Silva JAM, Alves LR, Silva MF, Camelo SHH, et al. Nursingsupervision for carecomprehensiveness. Revbrasenferm.[Internet] 2017 [acesso em 2018 mar 04]; 70(5): 1106- 
11.Disponível em: http://www.scielo.br/pdf/reben/v70n5/pt_0034-7167-reben-70-05-1106.pdf doi: 10.1590/0034-7167-2016-0491

4. Gomes RNS, Portela NLC, Pedrosa AO, Cunha JDS, Monte LRS, Santos CNC. Avaliação físicofuncional de salas de vacinas da rede pública municipal de Caxias-MA. Rev.pesqui. cuid. fundam. (Online). [Internet] 2016 [acesso em 2018 fev 21];8(1): 3793. Disponível em: http://www.indexf.com/pesquisa/2016/r83793.php doi: 10.9789/2175-5361.2016.v8i1.3793-3802

5. Kurcgant, P. Gerenciamento em Enfermagem. 1ํㅡㄹ edição. Rio de Janeiro. 2010.

6. Ministério da Saúde (BR). Secretaria de Vigilância em Saúde. Departamento de Vigilância das Doenças Transmissíveis. Manual de normas e procedimentos para vacinação. Brasília(DF): Ministério da Saúde; 2014.

7. Ministério da Saúde (BR). Portaria no 2.436, de 21 de setembro de 2017. Política Nacional de Atenção Básica. Estabelecendo a revisão de diretrizes para a organização da Atenção Básica, no âmbito do Sistema Único de Saúde (SUS). Diário Oficial da União. 22 Set 2017.

8. Oliveira VCD, Serrano GP, Gomes TS, Passos LMR, Pinto IC. Supervisão de enfermagem em sala de vacina: a percepção do enfermeiro. Texto \&contexto enferm. [Internet] 2013 [acesso em 2018 fev 12]; 22(4): 1015-21.Disponível em: http://www.scielo.br/pdf/tce/v22n4/18.pdf doi: 10.1590/S0104-07072013000400018.

9. Yin RK. Estudo de caso: planejamento e métodos. 5. ed. Porto Alegre: Bookman; 2015.

10. Instituto Brasileiro de Geografia e Estatística. Censo 2016 [acesso em 2017 ago. 20]. Disponível em: http://www.ibge.gov.br

11. Bardin, L. Análise de conteúdo. São Paulo: Edições. Vol 70. 2011.

12. Friese S, Conteras RB. "ATLAS. ti 8 user guide and reference." Berlin: ATLAS. ti Scientific Software Development GmbH. 2017.

13. Brasil.Conselho Nacional de Saúde. Resolução n. 466, de 12 de dezembro de 2012. Dispõe das diretrizes e normas regulamentadoras de pesquisas envolvendo seres humanos. Brasília(DF); 2012 dez 12 [acesso em 2018 abr 20]. Disponível em: http://conselho.saude.gov.br/resolucoes/2012/Reso466.pdf

14. Ximenes Neto FRG, Sampaio JJC. Processo de ascensão ao cargo e as facilidades e dificuldades no gerenciamento do território na Estratégia Saúde da Família. Rev Brás enferm.[Internet] 2008[acesso em 2017 out 26]; 61(1): 36-45. Disponível em: http://www.scielo.br/pdf/reben/v61n1/06.pdf doi: 10.1590/S003471672008000100006

15. Ceccim RB. Educação permanente em saúde: descentralização e disseminação de capacidade pedagógica na saúde. Ciênc Saúde Coletiva [internet]. 2005 [acesso em 19 de Jan. De 2019]; 10(4):975-86. Disponível em: http:// www.scielo.br/pdf/csc/v10n4/a20v10n4.pdf doi: 10.1590/S1413-81232005000400020 
16. Ximenes Neto FRG, Ferreira GB, Ximenes MRG, Costa SS, Negreiros JA. Necessidades de qualificação, dificuldades e facilidades dos técnicos de enfermagem na Estratégia Saúde da Família. Sanare-Revista de Políticas Públicas [inernet]. 2016. [acesso em 18 de Jun. de 2019] 15(1). Disponível em: https://sanare.emnuvens.com.br/sanare/article/view/927

17. Rodrigues AP, Santos FR, Abreu MO, Silva QRE, Pinto DRM. Boa gestão de enfermagem na percepção dos enfermeiros da estratégia de saúde da família. RevBras de Saúde Func.[Internet]2015 [acesso em 2018 fev 12]; 2(2): 12-22.Disponível em: http://www.seeradventista.com.br/ojs/index.php/RBSF/article/view/660/559

18. Gomes LMX, Barbosa TLDA, Silva CSO, Lopes JR, Leite MTDS. Prática gerencial do enfermeiro na Estratégia Saúde da Família. Trab. educ. saúde.[Internet]2015[acesso em 2017 out 22]; 13(3): 695707.Disponível em: http://www.scielo.br/pdf/tes/v13n3/1981-7746-tes-13-03-0695.pdf doi: 1590/1981-7746$\operatorname{sip} 00067$

19. Brasil. Ministério da Saúde. Resolução da Diretoria Colegiada - RDC no 197, de 26 de Dezembro de 2017 que dispõe sobre os requisitos mínimos para o funcionamento dos serviços de vacinação humana. Diário Oficial da União, Brasília, 28 Dez. 2017.

20. Marinelli NP, Posso MBS. Avaliação do dimensionamento espacial das unidades básicas de saúde. RevenfermUFPI.[Internet]2015[acesso em 2018 abr 12]; 4(1): 68-73.Disponível em: http://www.ojs.ufpi.br/index.php/reufpi/article/view/2324/pdf doi: 10.26694/reufpi.v4i1.2324

21. Schmitt MD, Maia JC, Almeida MDA, Adamy EK. Obstacles reported by nurses from the service of primary care in health at data collection. RevenfermUFPE.[Internet]2015[acesso em 2018 jan 09]; 9(3):7688-94.Disponível em: https://periodicos.ufpe.br/revistas/revistaenfermagem/article/view/10509/11385 doi: 10.5205/reuol.704961452-1-ED.0903supl201523.

22. Martins CCF, Santos VEP, Pereira MS, Santos NP. Relacionamento interpessoal da equipe de enfermagem x estresse: limitações para a prática. Cogitareenferm.[Internet] 2014[acesso em 2018 jan 08]; 19(2): 309-15. Disponível em: https://revistas.ufpr.br/cogitare/article/view/36985/22756 doi: 10.5380/ce.v19i2.36985.

23. Silva MP, Medeiros SM, Quental LLC. Relacionamento interpessoal da equipe de enfermagem: fragilidades e fortalezas. RevenfermUERJ.[Internet] 2016 [acesso em 2018 jan 21]; 24(5): e7657. Disponível em: http://www.facenf.uerj.br/v24n5/v24n5a09.pdf doi: 10.12957/reuerj.2016.7657.

24. Santiago ARJV, Cunha JXP. Supervisão de enfermagem: instrumento para a promoção da qualidade na assistência. Rev Saúde Pesqui.[Internet] 2011[acesso em 2018 jan 21];4(3): 443-8. Disponível em: http://periodicos.unicesumar.edu.br/index.php/saudpesq/article/view/1964/1395 doi: 10.12957/reuerj.2016.7657 
25. Pivoto FL, Filho WDL, Lunardi VL, Silva PA. Organização do trabalho e a produção de subjetividade da enfermeira relacionada ao processo de enfermagem. Esc Anna Nery RevEnferm.[Internet] 2017 [acesso em 2018 fev 03]; 21(1): e20170014. Disponível em: http://www.scielo.br/pdf/ean/v21n1/1414-8145-ean-21-01e20170014.pdf doi: 10.5935/1414-8145.20170014.

26. Carvalho ALRF. Supervisão clínica em enfermagem-estratégia para o conhecimento e qualidade dos cuidados. RevRENE.[Internet] 2017[acesso em 2018 jan 17];18(3): 291. Disponível em: periodicos.ufc.br/rene/article/download/20042/30693doi: 10.15253/2175-6783.2017000300001 .

27. Brasil. Ministério da Saúde. Secretaria de Vigilância em Saúde. Departamento de Vigilância Epidemiológica. (2004). Programa de avaliação do instrumento de supervisão sala de vacinação-PAISSV.

28. Zambiazi BRB, Costa AM. Gerenciamento de enfermagem em unidade de emergência: dificuldades e desafios. Revadmsaúde.[Internet]. 2013 [acesso em 2017 nov 12];15(61): 169-76. Disponível em: www.cqh.org.br/portal/pag/anexos/baixar.php?p_ndoc=1021\&p_nanexo=507

\section{Autor correspondente}

Heloiza Maria Siqueira Renno

E-mail: heloizarenno@gmail.com

Endereço: Rua Sebastião Gonçalves Coelho, 400 - Bairro Chanadour - Divinópolis, MG - Sala 207A

CEP: 35.501-296

\section{Contribuições de Autoria}

1 - Matheus Adriano Divino Pereira

Participou da concepção e planejamento do projeto de pesquisa, obtenção/análise e interpretação dos dados, redação e revisão crítica.

2 - Bruna Camargos de Lima

Participou da concepção e planejamento do projeto de pesquisa, obtenção/análise e interpretação dos dados, redação e revisão crítica.

3 - Deborah Amaral Donnini

Participou da concepção e planejamento do projeto de pesquisa, obtenção/análise e interpretação dos dados, redação e revisão crítica.

4 - Valéria Conceição de Oliveira

Participou da concepção e planejamento do projeto de pesquisa, obtenção/análise e interpretação dos dados, redação e revisão crítica. 
Gerenciamento de enfermagem em sala de vacina: desafios e potencialidades I 18

5 - Tarcísio Laerte Gontijo

Participou da concepção e planejamento do projeto de pesquisa, obtenção/análise e interpretação dos dados, redação e revisão crítica.

6 - Heloiza Maria Siqueira Renno

Participou da concepção e planejamento do projeto de pesquisa, obtenção/análise e interpretação dos dados, redação e revisão crítica.

\section{Como citar este artigo}

Pereira MAD, Lima BC, Donnini DA, Oliveira VC, Gontijo TL, Renno HMS. Gerenciamento de enfermagem em sala de vacina: desafios e potencialidades. Rev. Enferm. UFSM. 2019 [Acesso em: 2019 jun 15];vol 9 ex:1-18. DOI:https://doi.org/10.5902/2179769233279 